November 23, 2018 17:19 WSPC/Guidelines-IJMPA Casimir

International Journal of Modern Physics A

(C) World Scientific Publishing Company

\title{
CASIMIR DENSITIES FOR A MASSIVE FERMIONIC QUANTUM FIELD IN A GLOBAL MONOPOLE BACKGROUND WITH SPHERICAL BOUNDARY
}

\author{
A. A. SAHARIAN \\ Department of Physics, Yerevan State University \\ 375049 Yerevan, Armenia \\ E. R. BEZERRA DE MELLO \\ Departamento de Física-CCEN, Universidade Federal da Paraíba \\ 58.059-970, J. Pessoa, PB C. Postal 5.008, Brazil \\ Received (Day Month Year) \\ Revised (Day Month Year)
}

\begin{abstract}
We investigate the vacuum expectation value of the energy-momentum tensor associated with a massive fermionic field obeying the MIT bag boundary condition on a spherical shell in the global monopole spacetime. The asymptotic behavior of the vacuum densities is investigated near the sphere center and surface, and at large distances from the sphere. In the limit of strong gravitational field corresponding to small values of the parameter describing the solid angle deficit in global monopole geometry, the sphere-induced expectation values are exponentially suppressed.
\end{abstract}

Keywords: Global monopole; Casimir densities; fermionic field.

\section{Introduction}

Global monopoles are spherically symmetric topological defects created due to phase transition when a global symmetry is spontaneously broken and they have important role in the cosmology and astrophysics. The simplest theoretical model which provides global monopoles has been proposed by Barriola and Vilenkin. 1 Neglecting the small size of the monopole's core, the corresponding metric tensor can be approximately given by the line element

$$
d s^{2}=d t^{2}-d r^{2}-\alpha^{2} r^{2}\left(d \theta^{2}+\sin ^{2} \theta d \phi^{2}\right),
$$

where the parameter $\alpha^{2}$, smaller than unity, depends on the symmetry breaking energy scale and codifies the presence of the global monopole. This spacetime corresponds to an idealized point-like global monopole. Here we shall calculate the vacuum expectation values of the energy-momentum tensor for fermionic fields obeying MIT bag boundary condition on the spherical shell concentric with the global monopole. 


\section{Vacuum Energy-Momentum Tensor Inside a Spherical Shell}

The dynamics of a massive spinor field on a curved spacetime is described by the Dirac equation

$$
i \gamma^{\mu}\left(\partial_{\mu}+\Gamma_{\mu}\right) \psi-M \psi=0
$$

where $\gamma^{\mu}$ are the Dirac matrices and $\Gamma_{\mu}=\frac{1}{4} \gamma_{\nu} \nabla_{\mu} \gamma^{\nu}$ is the spin connection with $\nabla_{\mu}$ being the standard covariant derivative operator. Our interest in this paper will be the vacuum expectation values of the energy-momentum tensor induced by a spherical shell in the global monopole spacetime. We shall assume that on the sphere surface the field satisfies bag boundary condition:

$$
\left(1+i \gamma^{\mu} n_{b \mu}\right) \psi=0, \quad r=a,
$$

where $a$ is the sphere radius, $n_{b \mu}$ is the outward-pointing normal to the boundary. Expanding the field operator in terms of the complete set of single-particle states $\left\{\psi_{\beta}^{(+)}, \psi_{\beta}^{(-)}\right\}$and making use the standard anticommutation relations, for the vacuum expectation values of the energy-momentum tensor one finds the following mode-sum formula

$$
\left\langle 0\left|T_{\mu \nu}\right| 0\right\rangle=\sum_{\beta} T_{\mu \nu}\left\{\bar{\psi}_{\beta}^{(-)}(x), \psi_{\beta}^{(-)}(x)\right\},
$$

where $|0\rangle$ is the amplitude for the corresponding vacuum. For the geometry under consideration the eigenfunctions are specified by the set of quantum numbers $\beta=(k j m \sigma)$, where $j=1 / 2,3 / 2, \ldots$ determines the value of the total angular momentum, $m=-j, \ldots, j$ is its projection, and $\sigma=0,1$ corresponds to two types of eigenfunctions with different parities. These functions have the form

$$
\begin{aligned}
& \psi_{\beta}^{( \pm)}=A_{\sigma} \frac{e^{-i \omega t}}{\sqrt{r}}\left(\begin{array}{c}
Z_{\nu_{\sigma}}(k r) \Omega_{j l m} \\
i n_{\sigma} Z_{\nu_{\sigma}+n_{\sigma}}(k r) \frac{k(\hat{n} \cdot \vec{\sigma})}{\omega+M} \Omega_{j l m}
\end{array}\right), \quad n_{\sigma}=(-1)^{\sigma}, \\
& \omega= \pm E, \quad E=\sqrt{k^{2}+M^{2}}, \quad \nu_{\sigma}=\frac{j+1 / 2}{\alpha}-\frac{n_{\sigma}}{2}
\end{aligned}
$$

where $\hat{n}=\vec{r} / r, \vec{\sigma}=\left(\sigma^{1}, \sigma^{2}, \sigma^{3}\right)$ with the curved space Pauli $2 \times 2$ matrices $\sigma^{k}$. In Eq. (5) $Z_{\nu}(x)$ represents a cylindrical function of the order $\nu$ and $\Omega_{j l m}$ are the standard spinor spherical harmonics 2 with $l=j-n_{\sigma} / 2$.

For the region inside a spherical shell one has $Z_{\nu}(x)=J_{\nu}(x)$, where $J_{\nu}(x)$ is the Bessel function. The imposition of the boundary condition on the eigenfunctions (5) leads to the following equations for the eigenvalues

$$
\tilde{J}_{\nu_{\sigma}}(k a)=0
$$

where for a given function $F(z)$ we use the notation

$$
\tilde{F}(z) \equiv z F^{\prime}(z)+\left(\mu+s_{\omega} \sqrt{z^{2}+\mu^{2}}-(-1)^{\sigma} \nu\right) F(z), \quad \sigma=0,1,
$$

with $s_{\omega}=\operatorname{sgn}(\omega)$ and $\mu=M a$. Let us denote by $\lambda_{\nu_{\sigma}, s}=k a, s=1,2, \ldots$, the roots to equation (7) in the right half plane, arranged in ascending order. By using 
the standard integral for the Bessel functions, for the normalization coefficient one finds

$$
A_{\sigma}^{2}=\frac{z}{2 \alpha^{2} a^{2}} \frac{\sqrt{z^{2}+a^{2} M^{2}}+a M}{\sqrt{z^{2}+a^{2} M^{2}}} T_{\nu_{\sigma}}(z), \quad z=\lambda_{\nu_{\sigma}, s},
$$

with

$$
T_{\nu}^{-1}(z)=\frac{J_{\nu}^{2}(z)}{z}\left[z^{2}+\left(\mu-(-1)^{\sigma} \nu\right)\left(\mu+s_{\omega} \sqrt{z^{2}+\mu^{2}}\right)-\frac{s_{\omega} z^{2}}{2 \sqrt{z^{2}+\mu^{2}}}\right] .
$$

Because the spacetime is spherically symmetric and static it follows that the vacuum energy-momentum tensor is diagonal:

$$
\left\langle 0\left|T_{\mu}^{\nu}\right| 0\right\rangle=\operatorname{diag}\left(\varepsilon,-p,-p_{\perp},-p_{\perp}\right),
$$

with the energy density $\varepsilon$, radial, $p$, and azimuthal, $p_{\perp}$, pressures being functions on the radial coordinate only. As a consequence of the continuity equation $\nabla_{\nu}\left\langle 0\left|T_{\mu}^{\nu}\right| 0\right\rangle=0$, these functions are related by the equation

$$
r \frac{d p}{d r}+2\left(p-p_{\perp}\right)=0,
$$

which means that the radial dependence of the radial pressure necessarily leads to the anisotropy in the vacuum stresses. Below we will give formulas for $\varepsilon$ and $p$. The azimuthal pressure is expressed via the radial one by using formula (12).

Substituting eigenfunctions (5) into Eq. (4), the summation over the quantum number $m$ can be done by using standard summation formula for the spherical harmonics. For the energy-momentum tensor components one finds

$$
\begin{aligned}
q(r) & =\frac{-1}{8 \pi \alpha^{2} a^{3} r} \sum_{j=1 / 2}^{\infty}(2 j+1) \sum_{\sigma=0,1} \sum_{s=1}^{\infty} T_{\nu_{\sigma}}\left(\lambda_{\nu_{\sigma}, s}\right) f_{\sigma \nu_{\sigma}}^{(q)}\left[\lambda_{\nu_{\sigma}, s}, J_{\nu_{\sigma}}\left(\lambda_{\nu_{\sigma}, s} r / a\right)\right], \\
q & =\varepsilon, p
\end{aligned}
$$

where we have introduced the notations

$$
\begin{aligned}
& f_{\sigma \nu}^{(\varepsilon)}\left[z, J_{\nu}(y)\right]=z\left[\left(\sqrt{z^{2}+\mu^{2}}-\mu\right) J_{\nu}^{2}(y)+\left(\sqrt{z^{2}+\mu^{2}}+\mu\right) J_{\nu+n_{\sigma}}^{2}(y)\right], \\
& f_{\sigma \nu}^{(p)}\left[z, J_{\nu}(y)\right]=\frac{z^{3}}{\sqrt{z^{2}+\mu^{2}}}\left[J_{\nu}^{2}(y)-\frac{2 \nu+n_{\sigma}}{y} J_{\nu}(y) J_{\nu+n_{\sigma}}(y)+J_{\nu+n_{\sigma}}^{2}(y)\right] .
\end{aligned}
$$

Applying to the sums over $s$ the generalized Abel-Plana summation formula ${ }^{3}$, the components of the vacuum energy-momentum tensor can be presented in the form

$$
q(r)=q_{m}(r)+q_{b}(r), \quad q=\varepsilon, p, p_{\perp},
$$

where $q_{m}(r)$ does not depend on the radius of the sphere $a$ and is the contribution due to unbounded global monopole spacetime. The corresponding quantities for the massless case are investigated in Ref. 4. The second term on the right of formula 
(16) is induced by the presence of the spherical shell and can be presented in the form

$$
q_{b}(r)=\frac{1}{\pi^{2} \alpha^{2} a^{3} r} \sum_{l=1}^{\infty} l \int_{\mu}^{\infty} \frac{x^{3} d x}{\sqrt{x^{2}-\mu^{2}}} \frac{W\left[I_{\nu}(x), K_{\nu}(x)\right]}{W\left[I_{\nu}(x), I_{\nu}(x)\right]} F_{\nu}^{(q)}\left[x, I_{\nu_{\sigma}}(x r / a)\right],
$$

with

$$
\begin{aligned}
& F_{\nu}^{(\varepsilon)}\left[x, I_{\nu}(y)\right]=\left(1-\frac{\mu^{2}}{x^{2}}\right)\left\{I_{\nu-1}^{2}(y)-I_{\nu}^{2}(y)-\mu \frac{I_{\nu-1}^{2}(y)+I_{\nu}^{2}(y)}{W\left[I_{\nu}(x), K_{\nu}(x)\right]}\right\}, \\
& F_{\nu}^{(p)}\left[x, I_{\nu}(y)\right]=I_{\nu-1}^{2}(y)-I_{\nu}^{2}(y)-\frac{2 \nu-1}{y} I_{\nu}(y) I_{\nu-1}(y) .
\end{aligned}
$$

Here and below $l=j+1 / 2, \nu \equiv \nu_{1}=l / \alpha+1 / 2$, and for given functions $f(x)$ and $g(x)$ we use the notation

$$
\begin{aligned}
W[f(x), g(x)] & =\left[x f^{\prime}(x)+(\mu+\nu) f(x)\right]\left[x g^{\prime}(x)+(\mu+\nu) g(x)\right] \\
& +\left(x^{2}-\mu^{2}\right) f(x) g(x) .
\end{aligned}
$$

It can be easily checked that for a massless spinor field the boundary-induced part of the vacuum energy-momentum tensor is traceless and the trace anomalies are contained only in the purely global monopole part without boundaries.

Now we turn to the consideration of various limiting cases of the expressions for the sphere-induced vacuum expectation values. In the limit $r \rightarrow 0$, for the boundary parts (17) the summands with a given $l$ behave as $r^{2 l / \alpha-2}$, and the leading contributions come from the lowest $l=1$ terms. Making use standard formulae for the Bessel modified functions for small values of the argument, for the sphere-induced parts near the center, $r \ll a$, one finds

$$
\begin{aligned}
\varepsilon_{b} & \approx \frac{\pi^{-2} a^{-4}(r / 2 a)^{\frac{2}{\alpha}-2}}{2 \alpha^{2} \Gamma^{2}\left(\frac{1}{\alpha}+\frac{1}{2}\right)} \int_{\mu}^{\infty} d x x^{\frac{2}{\alpha}} \sqrt{x^{2}-\mu^{2}} \frac{W\left[I_{\nu}(x), K_{\nu}(x)\right]-\mu}{W\left[I_{\nu}(x), I_{\nu}(x)\right]}, \\
p_{b} & \approx \frac{\pi^{-2} a^{-4}(r / 2 a)^{\frac{2}{\alpha}-2}}{2 \alpha(2+\alpha) \Gamma^{2}\left(\frac{1}{\alpha}+\frac{1}{2}\right)} \int_{\mu}^{\infty} \frac{x^{\frac{2}{\alpha}+2} d x}{\sqrt{x^{2}-\mu^{2}}} \frac{W\left[I_{\nu}(x), K_{\nu}(x)\right]}{W\left[I_{\nu}(x), I_{\nu}(x)\right]},
\end{aligned}
$$

where $\nu=1 / \alpha+1 / 2$ and $\Gamma(x)$ is the gamma function. Hence, at the sphere center the boundary parts vanish for the global monopole spacetime $(\alpha<1)$ and are finite for the Minkowski spacetime $(\alpha=1)$. Note that in the large mass limit, $\mu \gg 1$, the integrals in Eqs. (21), (22) are exponentially suppressed by the factor $e^{-2 \mu}$.

The boundary induced parts of the vacuum energy-momentum tensor components diverge at the sphere surface, $r \rightarrow a$. In order to find the leading terms of the corresponding asymptotic expansion in powers of the distance from the sphere surface, we note that in the limit $r \rightarrow a$ the sum over $l$ in (17) diverges and, hence, for small $1-r / a$ the main contribution comes from the large values of $l$. Consequently, rescaling the integration variable $x \rightarrow \nu x$ and making use the uniform asymptotic expansions for the modified Bessel functions for large values of the order, to the leading order one finds

$$
\varepsilon_{b}(r) \approx-\frac{\mu+1 / 5}{12 \pi^{2} a(a-r)^{3}}, \quad p_{b}(r) \approx-\frac{1 / 5-2 \mu}{24 \pi^{2} a^{2}(a-r)^{2}} .
$$


Notice that the terms in these expansions diverging as the inverse fourth power of the distance have canceled out. This is a consequence of the conformal invariance of the massless fermionic field. It is of interest to note that the leading terms do not depend on the parameter $\alpha$ and, hence, are the same for the Minkowski and global monopole bulks. In Fig. [1 we have presented the dependence of the Casimir densities, $a^{4} q_{b}(r)$ on the rescaled radial coordinate $r / a$ for a massless spinor field on the Minkowski bulk (left panel) and on the global monopole background with the solid angle deficit parameter $\alpha=0.5$ (right panel). The vacuum energy density and pressures are negative inside the sphere.
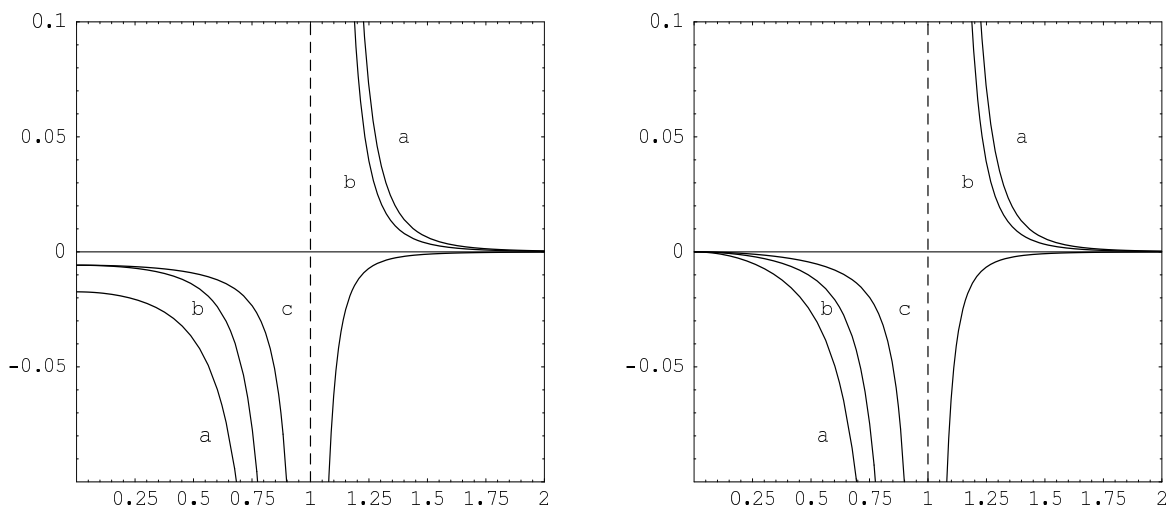

Fig. 1. Vacuum energy density, $a^{4} \varepsilon$ (curve a), azimuthal pressure $a^{4} p_{\perp}$ (curve b), and radial pressure $a^{4} p$ (curve c) for a massless spinor as functions on the ratio $r / a$ inside and outside a spherical shell in the Minkowski spacetime $(\alpha=1$, left panel) and for the global monopole spacetime with $\alpha=0.5$ (right panel).

Now let us consider the limit $\alpha \ll 1$ for a fixed value $r<a$. This limit corresponds to strong gravitational fields. In this case one has $\nu \approx l / \alpha \gg 1$. The main contribution to the sum over $l$ comes from the summands with $l=1$ and the boundary parts of the vacuum energy-momentum tensor components behave as $\exp [-2 \ln (a / r) / \alpha]$ with $p_{b} / p_{\perp b} \sim \alpha$. Hence, for $\alpha \ll 1$ the boundary-induced vacuum expectation values are exponentially suppressed and the corresponding vacuum stresses are strongly anisotropic. Fig. 2 shows that the nonzero mass can essentially change the behavior of the vacuum energy-momentum tensor components. In this figure we have depicted the dependence of the boundary induced quantities $a^{4} q_{b}(r)$ on the parameter $M a$ for the radial coordinate $r=0.5 a$. The left panel corresponds to the sphere in the Minkowski spacetime $(\alpha=1)$ and for the right panel $\alpha=0.5$.

\section{Vacuum Expectation Values Outside a Spherical Shell}

Now let us consider the expectation values of the energy-momentum tensor in the region outside a spherical shell, $r>a$. The corresponding eigenfunctions have the 

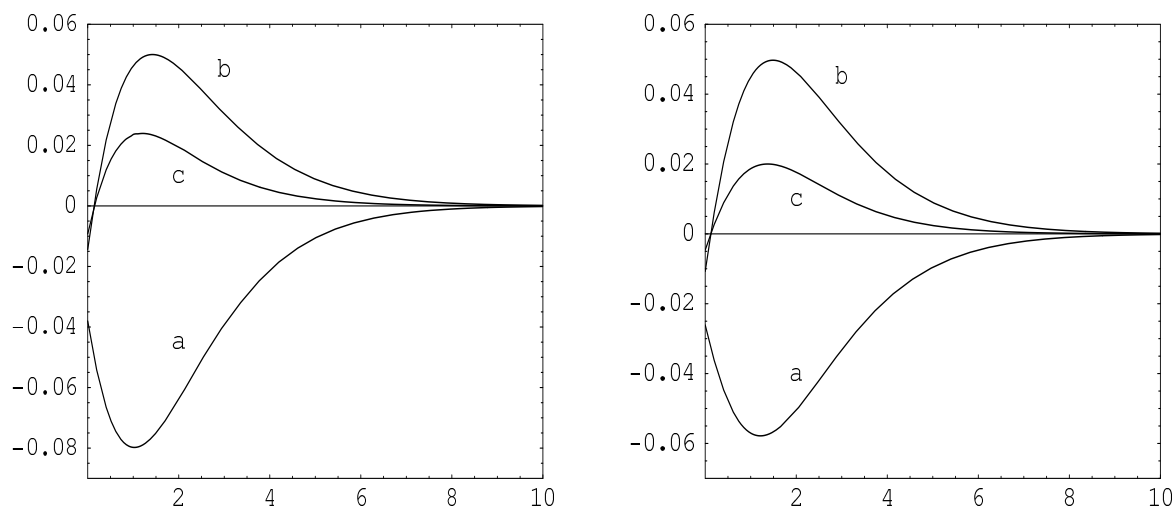

Fig. 2. Boundary-induced vacuum expectation values $a^{4} q_{b}(r), q=\varepsilon, p, p_{\perp}$, as functions on $\mu=$ $M a$ for $r / a=0.5$. The curves a, b, c correspond to the energy density $(\varepsilon)$, azimuthal pressure $\left(p_{\perp}\right)$, and radial pressure $(p)$, respectively. For the left panel $\alpha=1$ (Minkowski spacetime) and for the right panel $\alpha=0.5$.

form (15) with

$$
Z_{\nu}(k r)=g_{\nu}(k a, k r) \equiv J_{\nu}(k r) \tilde{Y}_{\nu}(k a)-Y_{\nu}(k r) \tilde{J}_{\nu}(k a),
$$

where $Y_{\nu}(z)$ is the Neumann function, and the functions with tilda are defined as (8). Now the spectrum for the quantum number $k$ is continuous and for the normalization coefficient we obtain

$$
A_{\sigma}^{2}=\frac{k(\omega+M)}{2 \alpha^{2} \omega\left[\tilde{J}_{\nu_{\sigma}}^{2}(k a)+\tilde{Y}_{\nu_{\sigma}}^{2}(k a)\right]} .
$$

Substituting the eigenfunctions (5) into the mode-sum formula (4) and taking into account Eqs. (24) and (25), we can see that the vacuum energy-momentum tensor has the form (11). The diagonal components are determined by formulae

$$
q(r)=\frac{-1}{8 \pi \alpha^{2} a^{3} r} \sum_{j=1 / 2}^{\infty}(2 j+1) \sum_{\sigma=0,1} \int_{0}^{\infty} d x \frac{f_{\sigma \nu_{\sigma}}^{(q)}\left[x, g_{\nu_{\sigma}}(x, x r / a)\right]}{\widetilde{J}_{\nu_{\sigma}}^{2}(x)+\tilde{Y}_{\nu_{\sigma}}^{2}(x)}, \quad q=\varepsilon, p,
$$

where the expressions for $f_{\sigma \nu_{\sigma}}^{(q)}\left[x, g_{\nu_{\sigma}}(x, x r / a)\right]$ are obtained from formulae (14), (15) by replacements

$$
J_{\nu}(y) \rightarrow g_{\nu}(x, y), \quad J_{\nu+n_{\sigma}}(y) \rightarrow J_{\nu+n_{\sigma}}(y) \tilde{Y}_{\nu}(k a)-Y_{\nu+n_{\sigma}}(y) \tilde{J}_{\nu}(k a) .
$$

To find the parts in the vacuum expectation values of the energy-momentum tensor induced by the presence of the sphere we subtract the corresponding components for the monopole bulk without boundaries. After rotation of the integration contour and introducing the Bessel modified functions, for the boundary-induced parts one obtains

$$
q_{b}(r)=\frac{1}{\pi^{2} \alpha^{2} a^{3} r} \sum_{l=1}^{\infty} l \int_{\mu}^{\infty} \frac{x^{3} d x}{\sqrt{x^{2}-\mu^{2}}} \frac{W\left[I_{\nu}(x), K_{\nu}(x)\right]}{W\left[K_{\nu}(x), K_{\nu}(x)\right]} F_{\nu}^{(q)}\left[x, K_{\nu}(x r / a)\right] .
$$


Here the expressions for the functions $F_{\sigma \nu}^{(q)}\left[x, K_{\nu}(y)\right]$ are obtained from formulae (18), (19) by replacements $I_{\nu}(y) \rightarrow K_{\nu}(y)$ and $I_{\nu-1}(y) \rightarrow-K_{\nu-1}(y)$. As for the interior region, it can be seen that in the limit of strong gravitational fields, $\alpha \ll 1$, the boundary induced vacuum expectation values are exponentially suppressed by the factor $\exp [-(2 / \alpha) \ln (r / a)]$, an the corresponding vacuum stresses are strongly anisotropic: $p_{b} / p_{\perp b} \sim \alpha$.

In Fig. 1 we have plotted the dependence of the vacuum energy density and stresses on the radial coordinate for a massless spinor field outside a sphere on the Minkowski bulk (left panel) and on background of the global monopole spacetime with $\alpha=0.5$. As seen form these figures, the energy density and azimuthal pressure are positive outside a sphere, and the radial pressure is negative. The latter has the same sign as for the interior region.

For the case of a massless spinor the asymptotic behavior of boundary part (28) at large distances from the sphere can be obtained by introducing a new integration variable $y=x r / a$ and expanding the subintegrands in terms of $a / r$. The leading contribution for the summands with a given $l$ has an order $(a / r)^{2 \nu+4}$ and the main contribution comes from the $l=1$ term. The leading terms for the asymptotic expansions over $a / r$ can be presented in the form

$$
q_{b}(r) \approx \frac{1}{2^{\frac{2}{\alpha}} \pi a^{4}} \frac{\Gamma\left(\frac{1}{\alpha}+1\right) \Gamma\left(\frac{2}{\alpha}+\frac{3}{2}\right) f_{q}}{\left(4-\alpha^{2}\right)(2+\alpha) \Gamma^{3}\left(\frac{1}{\alpha}+\frac{1}{2}\right)}\left(\frac{a}{r}\right)^{\frac{2}{\alpha}+5},
$$

where

$$
f_{\varepsilon}=4 \frac{\alpha+1}{3 \alpha+2}, \quad f_{p}=-\frac{2 \alpha}{3 \alpha+2}, \quad f_{p_{\perp}}=1 .
$$

As for the interior components, the quantities (28) diverge at the sphere surface $r=a$. Near the surface the dominant contributions come from modes with large $l$ and by making use the uniform asymptotic expansions for the Bessel modified functions, the asymptotic expansions can be derived in powers of the distance from the sphere. The leading terms of these asymptotic expansions are determined by formulae

$$
\varepsilon_{b}(r) \sim \frac{1 / 5-5 \mu}{12 \pi^{2} a(r-a)^{3}}, \quad p_{b}(r) \sim-\frac{1 / 5-2 \mu}{24 \pi^{2} a^{2}(r-a)^{2}} .
$$

Recall that near the sphere the interior energy density is always negative. As we see, the leading terms for the radial pressure are the same for the regions outside and inside the sphere. For the azimuthal pressure these terms have opposite signs. In the case of the massless spinor field the same is true for the energy density.

\section{Concluding Remarks}

In this paper we have analyzed the fermionic Casimir densities induced by a spherical shell in a idealized point-like global monopole spacetime. The boundary-induced expectation values for the components of the energy-momentum tensor are given by 
formulae (17) and (28) for interior and exterior regions, respectively. These expressions diverge in a non-integrable manner as the boundary is approached. The energy density and azimuthal pressure vary, to leading order, as the inverse cube of the distance from the sphere, and near the sphere the azimuthal pressure has opposite signs for the interior and exterior regions. For a massless spinor the same is true for the energy density. The radial pressure varies as the inverse square of the distance and near the sphere has the same sign for exterior and interior regions. The leading terms of the corresponding asymptotic expansions near the sphere do not depend on the solid angle deficit parameter and are the same for these two cases. Near the sphere the interior energy density is negative for all values of the mass, while the exterior energy density is positive for $M a<0.04$ and is negative for $M a>0.04$. Near the sphere center the dominant contributions come from modes with $l=1$ and the sphere-induced vacuum expectation values vanish for the global monopole spacetime and are finite for the Minkowski bulk. At large distances from the sphere the components of the vacuum energy-momentum tensor go to zero as $(a / r)^{2 / \alpha+5}$. In the limit of strong gravitational field, corresponding to small values of the parameter $\alpha$, describing the solid angle deficit, the boundary-induced part of the vacuum energy-momentum tensor is strongly suppressed by the factor $\exp [-(2 / \alpha)|\ln (r / a)|]$

and the corresponding vacuum stresses are strongly anisotropic: $p_{b} \sim \alpha p_{\perp b}$. Note that this suppression effect also takes place in the scalar case. $[5$

\section{References}

1. M. Barriola and A. Vilenkin, Phys. Rev. Lett. 63, 341 (1989).

2. V. B. Berestetskii, E. M. Lifshits and L. P. Pitaevskii, Quantum Electrodynamics (Pergamon Press, Oxford, 1982).

3. A. A. Saharian, Report No. IC/2000; hep-th/0002239

4. E. R. Bezerra de Mello, V. B. Bezerra, and N. R. Khusnutdinov, Phys. Rev. D60, 063506 (1999).

5. A. A. Saharian and M. R. Setare, Class. Quantum Grav. 20, 3765 (2003). 